\title{
Behcet's disease with primary hypothyroidism, adrenal insufficiency and coeliac disease: A case report
}

\author{
Ameer Kakaje ${ }^{1}$ and Rama Awad ${ }^{2}$ \\ ${ }^{1}$ Damascus University Faculty of Medicine \\ ${ }^{2}$ Al-Mouwasat Hospital
}

April 28, 2020

\begin{abstract}
Behcet's disease (BD) is a vasculitis of unknown aetiology that is reported to accompany autoimmune diseases. We report a rare case of $\mathrm{BD}$, Addison's disease, Hashimoto's disease, and subclinical coeliac disease all occurring in one patient which has not previously been reported.
\end{abstract}

\section{Key clinical message :}

We are reporting a patient with Bechet's disease who had multiple autoimmune diseases that affected the thyroid, adrenal glands and had sub-clinical coeliac disease. It is controversial the correlation of Bechet's disease with autoimmune disease.

Keywords: Behcet's disease; hypothyroidism; Addison's disease; coeliac disease; Polyglandular autoimmune syndrome; Crohn's disease.

\section{Introduction:}

Behcet's disease (BD) is a type of vasculitis that manifests as periods of aggravations and improvements. It is a multi-system chronic inflammation that affects all vessels, particularly in the young patients [1]. It doesn't have a precise definition as it influences multiple organs and has unknown etiology [2]. We present you a BD patient with multiple-autoimmune anomalies.

\section{Case Report:}

A 21-year-old man came to the emergency room, complaining of nausea and recurrent vomiting for the past two days after any oral intake. He was afebrile and didn't recall eating something unusual. He also suffered for a month from malaise and dizziness when standing up. The dizziness improved while lying down. No loss of conscious, diarrhea or hematemesis was reported, but he had a history of BD. On examination, his blood pressure $(\mathrm{BP})$ was low $(60 \backslash 40 \mathrm{mmHg})$ and had a tachycardia (117 beats $/ \mathrm{min})$. The patient reported normal appetite and denied weight loss. He had a history of $\mathrm{BD}$ which was diagnosed 4 years ago. At that time, he had arthralgia and the triad of BD, which was recurrent oral aphthous and genital ulcers, and iritis. He was treated with cyclosporine and naproxen and the patient decided to stop the treatment 18 months ago. He denied taking cortisol and his surgical history was insignificant. He had a family history of Crohn's disease with his brother and rheumatoid arthritis with his sister. Hyperpigmentation was observed in the internal mucosa of the lips and gingiva (figure 1 ). Normal saline was infused and antiemetic was given intramuscularly. After stabilisation, his BP became $100 \backslash 70 \mathrm{mmHg}$ with normal heartbeats. His labs showed hyponatremia, mild hyperkalemia and normal glucose. ECG and chest x-ray were also normal. 8 AM cortisol was low with very high ACTH levels. Furthermore, TSH level was elevated, and TPO antibodies 
were positive. Thyroid ultrasonography showed a diffuse enlarged thyroid and radioactive iodine uptake test showed a low iodine uptake in the thyroid. This resulted in the diagnosis of HD. Anti TTG IgA was very high and duodenum biopsy showed stage 3a Marsh score which indicated CD.

\section{Discussion:}

BD lacks specific histological or laboratory findings [2] and is very polymorphous and commonly fatal. The patient had BD triad which is diagnostic according to ICBD 2014 criteria [3]. BD etiology and pathophysiology are controversial with conflicting research results; BD is believed to cause immunological disturbances which affect immune regulation, making AID more likely to occur [4]. Autoinflammatory origin is also suggested for BD [5], with the result that BD can resemble Crohn's disease by having polygenic autoinflammation where IL-1 $\beta$ increases during attacks [6]. This made the fact that the sister of the patient in our case had Crohn's disease seem relevant. In contrast, although BD typically causes hyper- vascularization to approximately all endocrine glands, an autoimmune etiology is suggested as the underline cause for BD rather than direct attacks from vasculitis [7]. There is also controversial data about thyroid functions (TSH, fT4, and T3) in BD patients. However, immunological mechanisms are suggested to be involved as autoreactive $\mathrm{T}$ cells play a major role in $\mathrm{BD}$ which may explain thyroid dysfunction in $\mathrm{BD}[8]$. Furthermore, few reports correlate $\mathrm{BD}$ with $\mathrm{AD}$. One suggested a partially dysfunctional adrenal glands [9]. In our case, the patient suffered from multiple AID which were $\mathrm{HD}, \mathrm{AD}$ and $\mathrm{CD}$.

When multiple endocrines are being targeted by the immune system in one individual, he may have polyglandular autoimmune syndrome (PGA). The most common type is PGA-ll which is when a patient has AD with either autoimmune thyroid disease or diabetes type 1 or both. It may correspond with other AID such as CD. This is termed in combination as Schmidt syndrome [10]. In our case, the patient did not carry out any genetic test and family history was negative for $\mathrm{HD}, \mathrm{AD}$, diabetes type $1, \mathrm{BD}$ and $\mathrm{CD}$ despite having 4 siblings in the family. This makes hereditary PGA-ll unlikely. Moreover, it is not evident whether ceasing the Immune-modulating drugs caused this AID in the BD patient, caused PGA-ll to be symptomatic, or was irrelevant.

In conclusion, we can neither confirm nor deny that our patient could have presented with PGA-ll and BD in unrelated manner. However, this is the first-reported case in that manner for best of our knowledge and further research is required.

Author contributions:

- Ameer Kakaje M.D: Drafting, literature check, writing and revision, and final submission.

- Rama Awad M.D: Acquiring and analysing the data and final revision of the text.

\section{References:}

1. Holl-Ulrich, K., [Vasculitis: New nomenclature of the Chapel Hill consensus conference 2012]. Z Rheumatol, 2014. 73 (9): p. 823-33; quiz 834-5.

2. Yazici, H., et al., Behçet syndrome: a contemporary view.Nature Reviews Rheumatology, 2018. 14 (2): p. 107-119.

3. Davatchi, F., et al., The International Criteria for Behçet's Disease (ICBD): a collaborative study of 27 countries on the sensitivity and specificity of the new criteria. Journal of the European Academy of Dermatology and Venereology, 2014. 28 (3): p. 338-347.

4. Yazici, H., The place of Behcet's syndrome among the autoimmune diseases. Int Rev Immunol, 1997. 14 (1): p. 1-10.

5. Gul, A., Behcet's disease as an autoinflammatory disorder.Curr Drug Targets Inflamm Allergy, 2005. 4 (1): p. 81-3.

6. Franks, W.A., et al., Cytokines in human intraocular inflammation. Curr Eye Res, 1992. 11 Suppl : p. 187-91. 
7. Bouomrani S, T.S., Guermazi M, Regaïeg N, Thyroid Involvement In Behçet's Disease. Symbiosis, 2019(Int J Thyro Disord Ther 1 (1): 1-7).

8. Lockwood, C.M., et al., Remission induction in Behcet's disease following lymphocyte depletion by the anti-CD52 antibody CAMPATH 1-H.Rheumatology (Oxford), 2003. 42 (12): p. 1539-44.

9. Colak, R., et al., A comparison between the effects of low (1 ug) and standard dose (250 ug) ACTH stimulation tests on adrenal cortex functions with Behcet's disease. Journal of the European Academy of Dermatology and Venereology, 2006. 20 (6): p. 721-725.

10. Husebye, E.S., M.S. Anderson, and O. Kampe, Autoimmune Polyendocrine Syndromes. N Engl J Med, 2018. 378 (12): p. 1132-1141.

Figure legends:

Figure 1 shows oral hyperpigmentation (orange arrows).

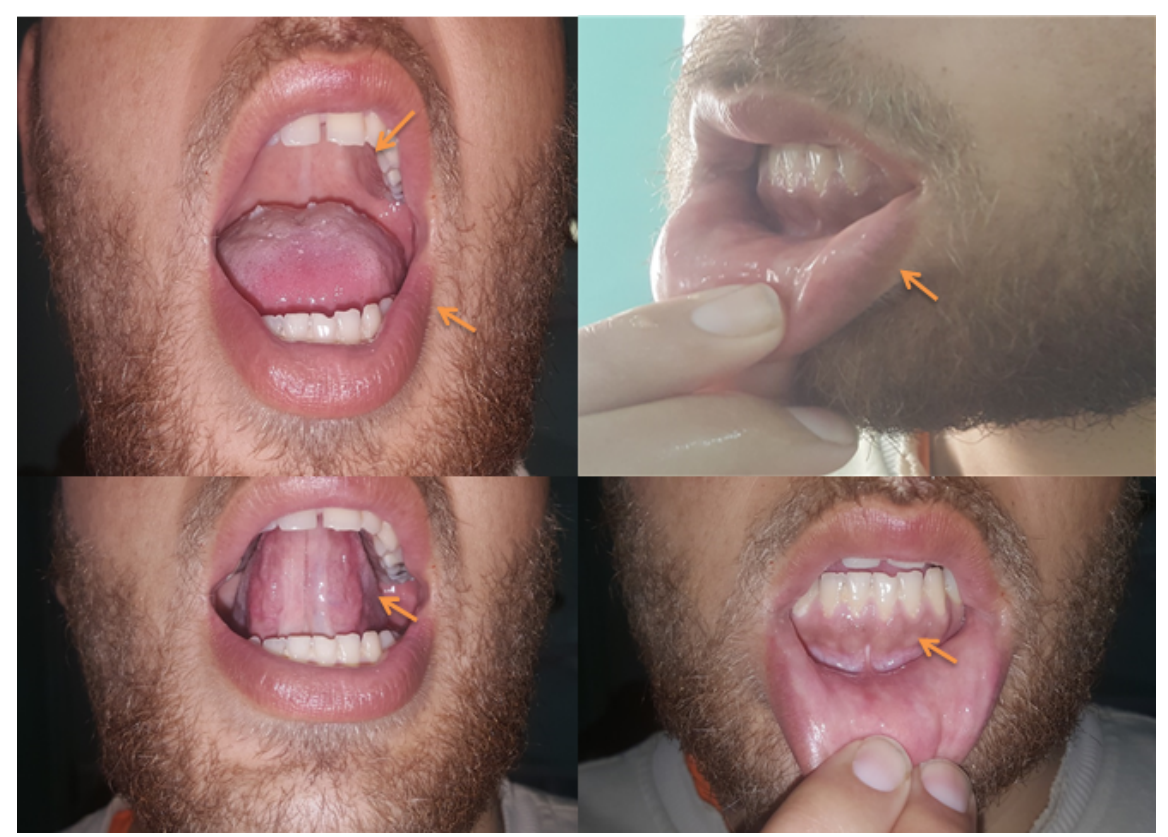

